\title{
UV Treatment of the Stabilizing Shell for Improving the Photostability of Silver Nanoparticles
}

\author{
Silvia Rinaldi, Luigi Tarpani, and Loredana Latterini \\ Dipartimento di Chimica, Biologia e Biotecnologie, Università degli Studi di Perugia, Via Elce di Sotto 8, 06123 Perugia, Italy \\ Correspondence should be addressed to Loredana Latterini; loredana.latterini@unipg.it
}

Received 21 July 2016; Accepted 9 August 2016

Academic Editor: Giovanni Bongiovanni

Copyright ( 2016 Silvia Rinaldi et al. This is an open access article distributed under the Creative Commons Attribution License, which permits unrestricted use, distribution, and reproduction in any medium, provided the original work is properly cited.

\begin{abstract}
Silver nanoparticles or nanoclusters are quite sensitive to light exposure. In particular, irradiation in the localized surface plasmon resonance (LSPR) region brings about a drastic modification of their optical properties due to growth and reshaping of the nanoparticles. In order to obtain luminescent colloids, small silver colloidal nanoparticles were prepared in chloroform using vinylpyrrolidone oligomers as capping agent and their luminescence properties were used to control their stability upon prolonged exposure to visible light. The polymeric shell around the metal clusters was hardened through photo-cross-linking by UV light. This process did not alter the morphology and the optical properties of the nanoparticles but greatly improved the particle photostability as confirmed also by confocal laser scanning microscopy measurements. The data clearly show that UV curing of the stabilizing layer could be a simple postsynthetic procedure to obtain materials with stable properties.
\end{abstract}

\section{Introduction}

Noble metal nanoparticles have attracted much attention in the last decades because they can be prepared through simple and versatile methods resulting in $\mathrm{nm}$ sized nanostructures whose dimension and shape finely tune their optical and electronic properties [1-3]. The nm sized particles show an intense optical response determined by the surface plasmon resonance (SPR), whose frequency and spectral broadening can be controlled by the shape, composition, and concentration of the colloidal nanomaterial $[4,5]$. It has been demonstrated that metal nanocrystals having subnanometer to $2 \mathrm{~nm}$ size (metal nanoclusters, NCs) show bright photoluminescence upon excitation with visible light of their colloidal samples. The energy and the efficiency of this radiative recombination process are strongly dependent on the size, shape, and environment of the NCs [6-9]. These optical properties together with their low toxicity, compared to other nanomaterials, make noble metal nanoparticles particularly challenging nanomaterials for biological applications such as colorimetric labelling or sensing $[10,11]$ and bioimaging in high resolution luminescence or surface-enhanced Raman scattering (SERS) spectroscopy [12-15]. However, it has been already reported that metal NCs show a strong tendency to growth and/or reshape upon prolonged irradiation due to their high surface energy $[16,17]$. The morphological changes induced by light generally result in alterations of their optical properties such as the luminescence or the LSPR useful to achieve the enhancement of Raman signals (the SERS effect). Hence, limitations may arise in the use of the silver NCs for optical and photonic applications due to their limited photostability in colloidal form.

Size-controlled templates such as dendrimers [18], DNA $[19,20]$, or zeolites $[9,21]$ have been exploited in the preparation of metal NCs in order to control their growth and stability. However, this approach might bring about additional issues concerning the postsynthesis processing of the metal cluster surfaces. Photoinduced polymerization (UV curing) of monomers or oligomers is one of the most efficient ways to prepare cross-linked polymer networks. In short times and selectively on the irradiated area, monomeric or oligomeric units can be transformed into viscous polymers which might be very resistant to environment conditions [22-24]. Considering the ability of some oligomers to undergo cross-linking reactions, a robust capping shell for the nanoparticles can be created by UV curing. Thus, this procedure has been successfully used to improve the stability and the optical properties of organic nanoparticles $[25,26]$. 


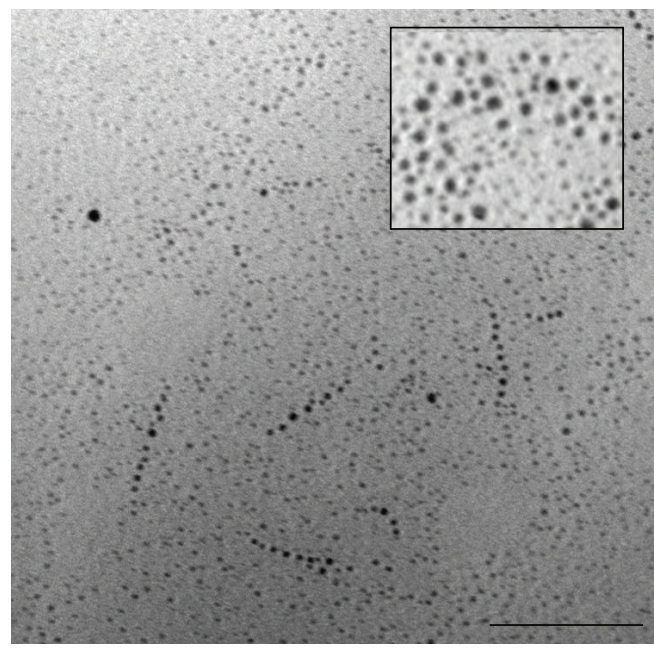

(a)

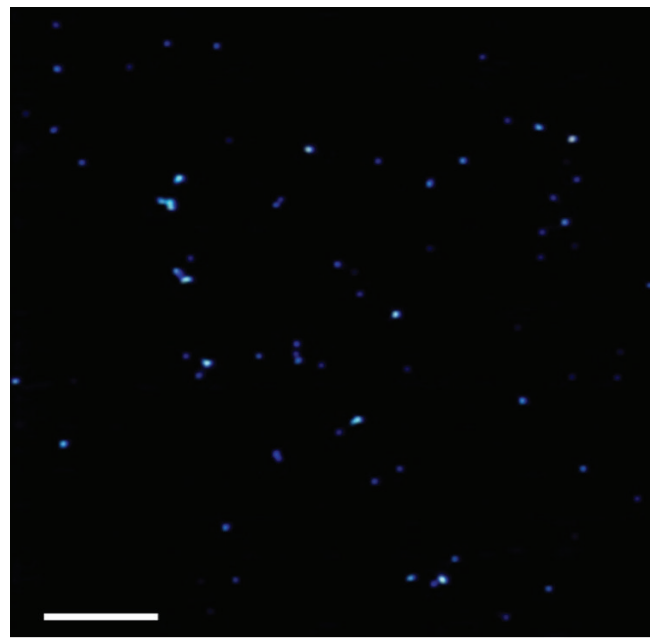

(c)

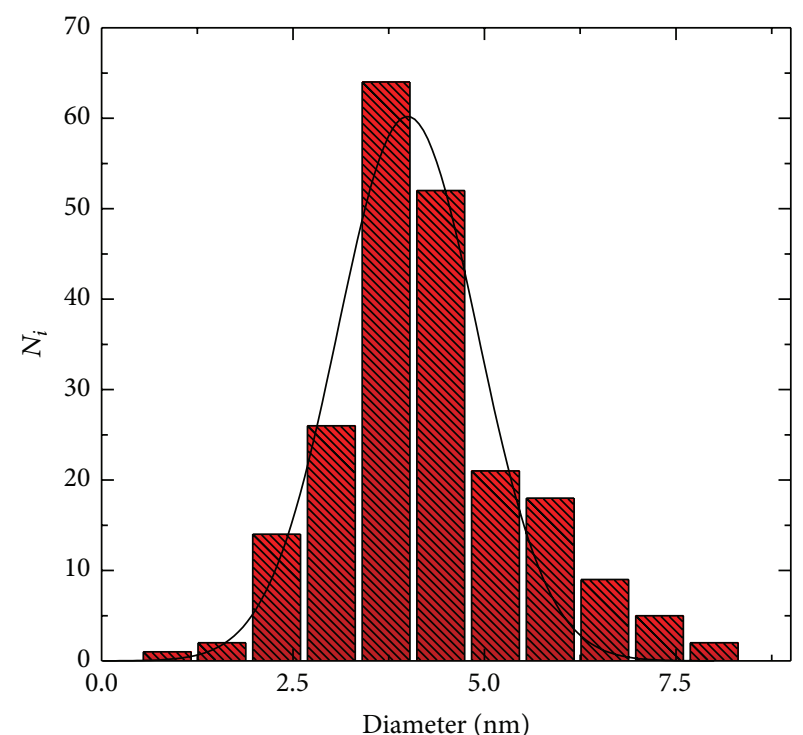

(b)

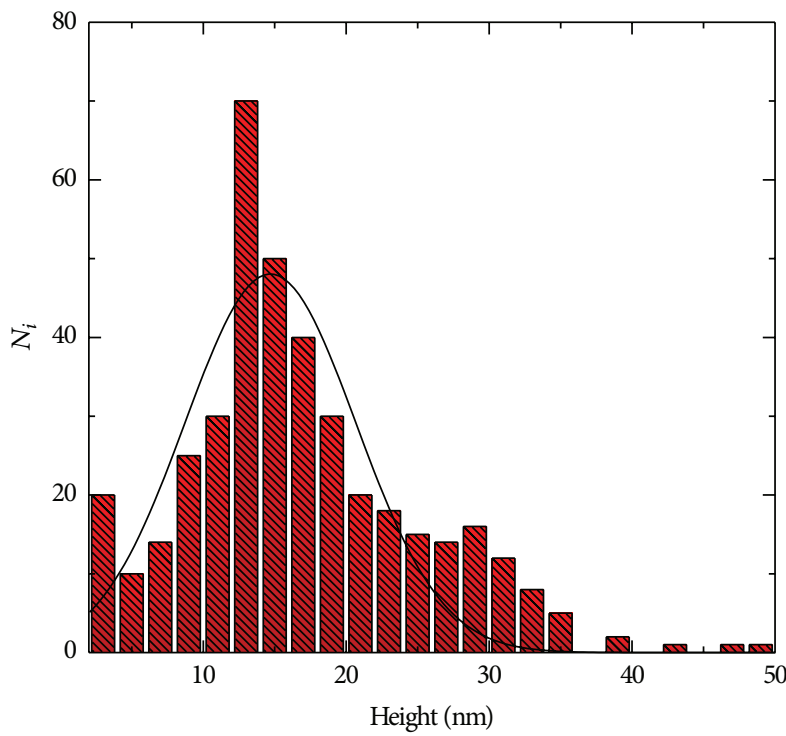

(d)

Figure 1: (a, b) TEM image and relative size distribution histogram of Ag-NCs (scale bar $=100 \mathrm{~nm}$; inset is $20 \times 20 \mathrm{~nm}$ zoom of (a)); (c, d) AFM image (scale bar $=500 \mathrm{~nm}$ ) of Ag-NCs and relative height distribution histograms.

In this work, we prepared luminescent silver nanoclusters (Ag-NCs) in an organic solvent by a fast and reliable reduction method and we demonstrated that photo-crosslinking of their polymeric stabilizing shell assures the photostabilization of the colloids and hence of their luminescence properties under prolonged light exposure. In this framework, UV curing of the nanoparticles surface resulted in an efficient method to obtain stable emitting materials for optical imaging applications.

\section{Materials and Methods}

2.1. Reagents. $\mathrm{AgNO}_{3}$ (Sigma-Aldrich, ACS reagent, $\geq 99.0 \%$ ), polyvinylpyrrolidone (PVP, Mw $=10 \mathrm{KDa}$, Sigma-Aldrich), and $\mathrm{CHCl}_{3}$ (99.4\% GC-grade) were used as received.
2.2. Synthesis of Silver Nanoparticles. $\mathrm{AgNO}_{3}(0.017 \mathrm{~g})$ was added to $100 \mathrm{~mL}$ of $\mathrm{CHCl}_{3}$ boiling solution containing $0.75 \mathrm{~g}$ of PVP. The solution was kept boiling at reflux for 30 minutes under vigorous stirring and then the resulting colloidal solution was cooled in an ice bath.

2.3. Morphological Characterization of Ag Colloids. A Philips mod. 208 TEM microscope (operating at $80 \mathrm{kV}$ of beam acceleration) was used to analyze the size distribution of the nanoparticles. The nanoparticles dissolved in $\mathrm{CHCl}_{3} / \mathrm{PVP}$ suspensions were deposited in 400-mesh carbon-coated copper support grids and left overnight in a desiccator to let the solvent evaporate. The size distribution histograms for the samples were obtained by analyzing 150-200 nanoparticles for each sample. An atomic force microscope (Solver-Pro 


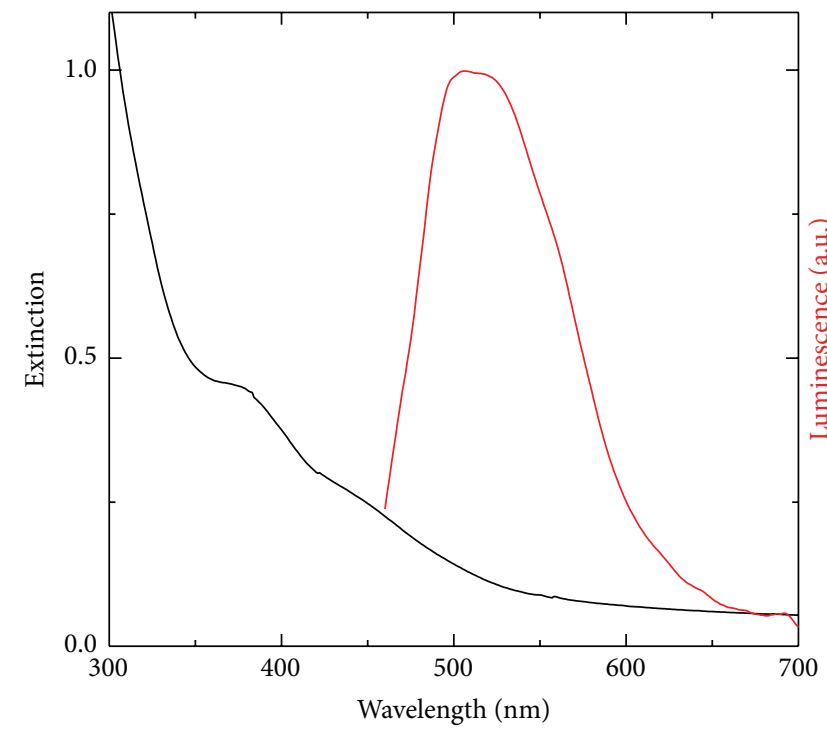

(a)

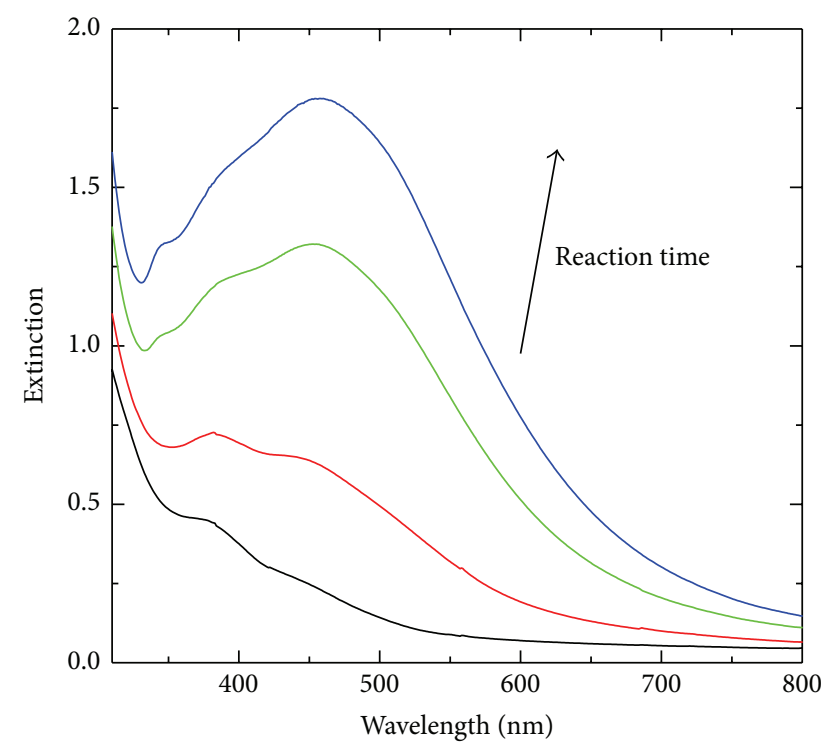

(b)

Figure 2: (a) Extinction and luminescence spectra $\left(\lambda_{\text {exc }}=450 \mathrm{~nm}\right)$ of Ag-NCs suspension in $\mathrm{CHCl}_{3}$; (b) extinction spectra of Ag-NCs suspension in $\mathrm{CHCl}_{3}$ at increasing reaction times $\left(30^{\prime}-5 \mathrm{~h}\right)$.

P47H, NT-MDT) was used to record topography images of the Ag-NCs. The measurements were carried out in semicontact conditions by the use of $190-325 \mathrm{kHz}$ cantilevers. A drop of the sample suspended in $\mathrm{CHCl}_{3}$ was placed on a mica substrate and spin-coated in order to spread the particles during solvent evaporation. A grain analysis was carried out to determine the size distribution of the particles from the images. The size distribution histograms for the samples were obtained by analyzing 150-200 nanoparticles for each sample.

2.4. Optical Characterization of Ag Colloids. UV-Vis absorption spectra of the $\mathrm{Ag}$ suspensions were recorded with a double beam Perkin-Elmer Lambda 800 spectrophotometer. Corrected luminescence spectra were acquired with a Fluorolog-2 (Spex, F112AI) fluorimeter. The luminescence images were recorded through a Nikon PCM2000 laser scanning confocal microscope, using a diode laser $\left(\lambda_{\text {exc }}=400 \mathrm{~nm}\right)$ as light source and selecting the emission spectral region by a band-pass filter $(520 \pm 30 \mathrm{~nm})$. The images were obtained with a $60 x 1.4$ N.A. oil immersion objective $(512 \times 512$ pixels $)$.

\section{Results and Discussion}

Silver nanocrystals (Ag-NCs) were prepared in $\mathrm{CHCl}_{3}$ starting from $\mathrm{AgNO}_{3}$ and using polyvinylpyrrolidone oligomers (PVP) as stabilizing agent. Although a similar preparation method has been already developed using DMF as a solvent $[27,28]$, to the best of our knowledge, this is the first report of the formation of PVP-stabilized Ag-NCs in chloroform. The PVP role is actually more complicated since, besides acting as stabilizer, it interacts with the $\mathrm{Ag}^{+}$ions producing organic radicals, which promote the silver reduction and the formation of the nanoclusters in the organic solvent [29]. In our preparation, the concentration of PVP was optimized to reach good quantitative coordination of $\mathrm{Ag}^{+}$, an efficient nucleation process, and good control of the growth process of $\mathrm{Ag}$ colloids. In particular, high $\mathrm{PVP} / \mathrm{Ag}^{+}$concentration ratios were chosen to ensure efficient transfer of silver ions into the organic solvent, thus achieving a fast nucleation rate. Furthermore, a homogeneous shell of the stabilizing polymer around the metal cores prevents the anisotropic growth and the development of nonspherical nanostructures, as reported in the literature $[30,31]$. As the reaction proceeded at the boiling temperature of $\mathrm{CHCl}_{3}$, the solution turned from colorless to transparent pale yellow; after 30 minutes, the resulting colloidal solution was cooled in an ice bath and morphologically and optically characterized.

TEM imaging was used to determine the size and morphology of the samples. Figure 1(a) shows that quasi-spherical silver nanoparticles were obtained in the chosen experimental conditions. Size distribution histograms (Figure 1(b)) were built from TEM images and analyzed by Gaussian functions. The analysis showed that the nanoparticles had a mean diameter of $2.9 \pm 0.5 \mathrm{~nm}$, thus indicating that a fraction of the colloids had dimensions below $2 \mathrm{~nm}$ range. AFM images (Figure 1(c)) allowed accessing size information on the entire particles (metal core and polymer shell) thus to estimate the dimension of the stabilizer shell. AFM-height distribution histograms (Figure 1(d)) could be reproduced by a Gaussian function, which was centered at $13.9 \pm 5.1 \mathrm{~nm}$. This broad width suggests that the stabilizer shell might have different arrangements around the silver cores. By comparing the nanoparticle dimensions determined from TEM and AFM measurements, an average PVP shell thickness of $11 \mathrm{~nm}$ was estimated. This value is in agreement with literature data for similar systems [32] in which a strong steric capping effect is desirable to control the particle growth.

An extinction spectrum with band-edge features at wavelength below $400 \mathrm{~nm}$ was recorded for the Ag-NC suspensions (Figure 2(a)). The spectrum does not resemble 

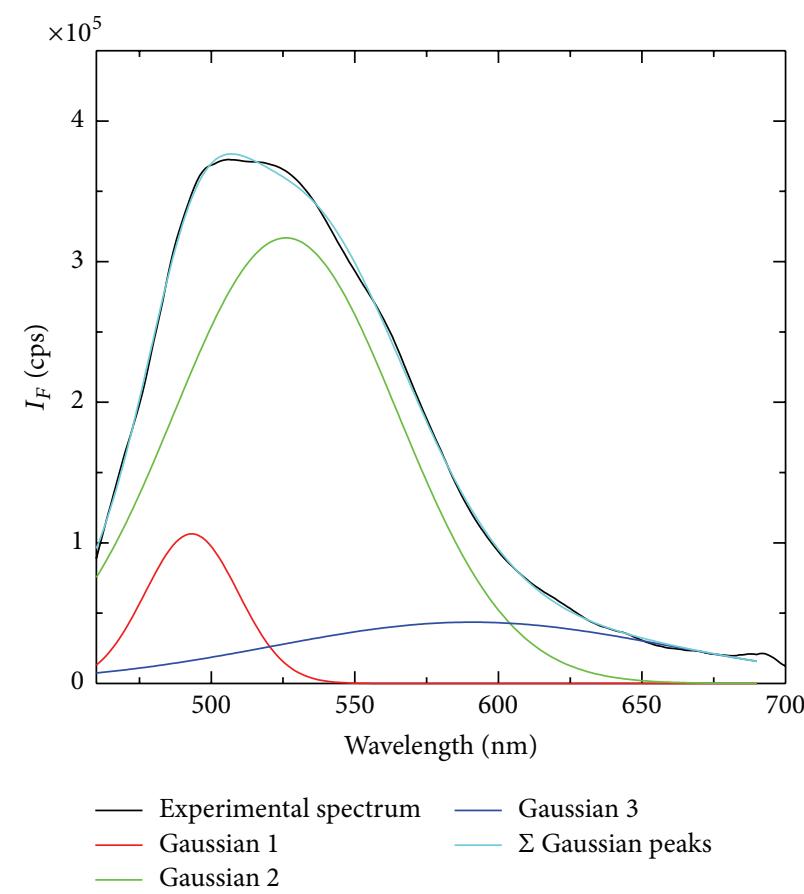

(a)

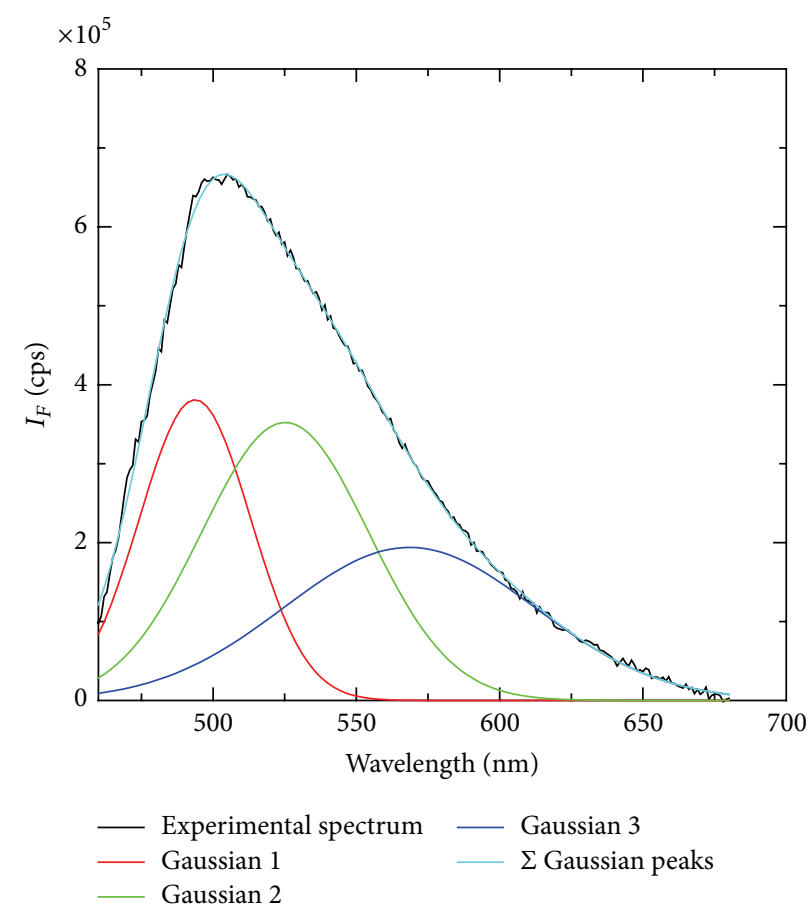

(b)

FIGURE 3: Luminescence spectra of (a) untreated and (b) photo-cross-linked Ag-NCs in $\mathrm{CHCl}_{3}\left(\lambda_{\text {exc }}=450 \mathrm{~nm}\right)$ together with their convolution with Gaussian functions.

the one reported for plasmonic silver nanoparticles [33], which could be achieved, in the synthetic conditions used, only by prolonging the reaction time to hours (Figure 2(b)).

The emission of the Ag-NCs colloidal suspension was observed upon excitation at $450 \mathrm{~nm}$ (Figure 2(a)). The luminescence spectrum was centered at $510 \mathrm{~nm}$ and presented a quite broad shape $\left(\mathrm{FWHM}=7500 \mathrm{~cm}^{-1}\right)$ likely reflecting the size distribution histogram (Figure 1(b)). The broad emission spectrum could be successfully fitted by the convolution of three Gaussian curves centered at 493, 526, and $590 \mathrm{~nm}$, respectively, supporting the presence of different NC populations (Figure 3(a)).

However, the luminescent properties of the colloidal suspension changed when it was subjected to prolonged Vis light exposure (at $420 \mathrm{~nm}$, light dose higher than $0.1 \mathrm{~J} / \mathrm{cm}^{2}$ ), since a progressive decrease of the emission intensity was observed (Figure 4). The occurrence of these quenching phenomena could be explained considering that the Vis light is able to induce Ostwald ripening processes in the silver suspensions. This is able to change dimensions and shapes of silver colloids, as previously reported in the literature $[16,17]$. TEM images of the colloids after irradiation (inset in Figure 4) revealed the formation of metal particles whose size is bigger compared to the pristine ones and whose shape markedly deviates from the spherical one. Thus, the luminescence decrease can be explained by the photoinduced growth of the nanoparticles [16].

To improve the photostability of the as-prepared Ag-NCs, selective photo-cross-linking of the polymeric shell has been

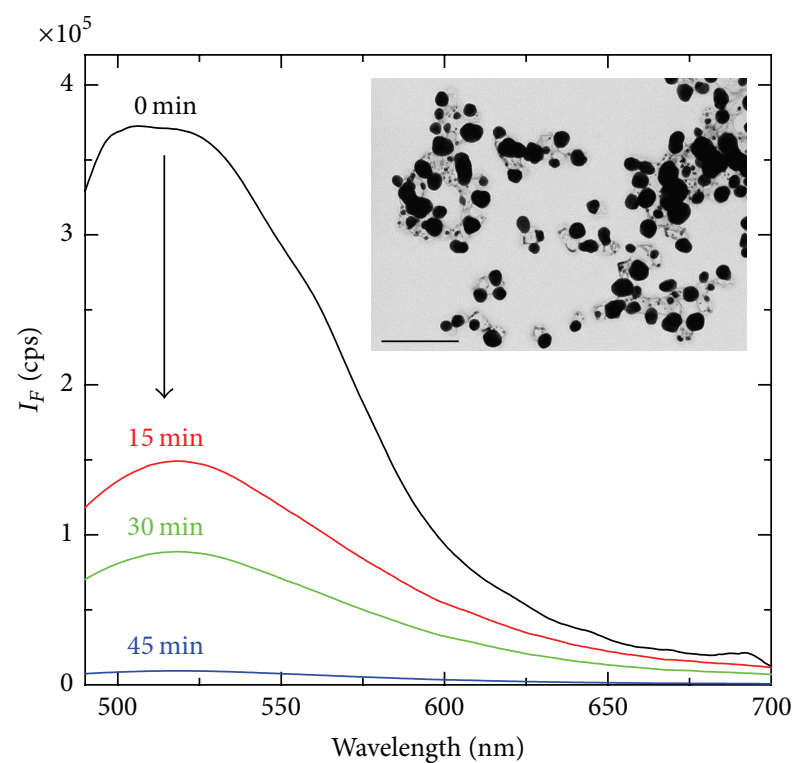

FIGURE 4: Luminescence spectra of the untreated Ag-NCs in $\mathrm{CHCl}_{3}$ $\left(\lambda_{\text {exc }}=450 \mathrm{~nm}\right)$ at different irradiation times $(0-45 \mathrm{~min})$ at $420 \mathrm{~nm}$. Inset is a TEM image of the Ag-NCs after irradiation (scale bar = $500 \mathrm{~nm})$.

induced by irradiation at $254 \mathrm{~nm}$, where PVP performs absorption efficiently. As said above, under UV light, vinylpyrrolidone is able to cross-link, conferring to the environment an increased rigidity [34]. According to the literature 


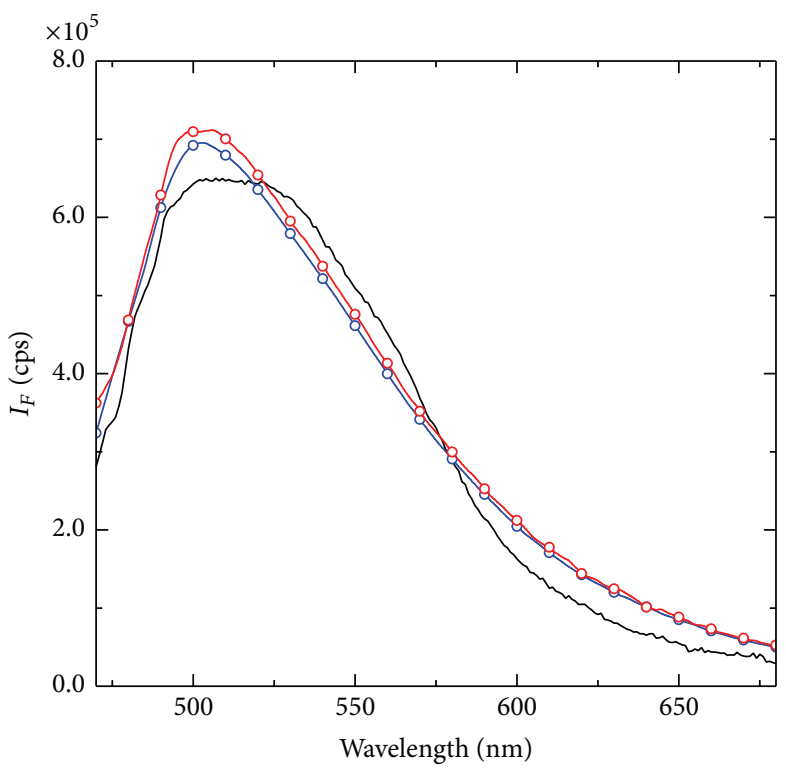

(a)

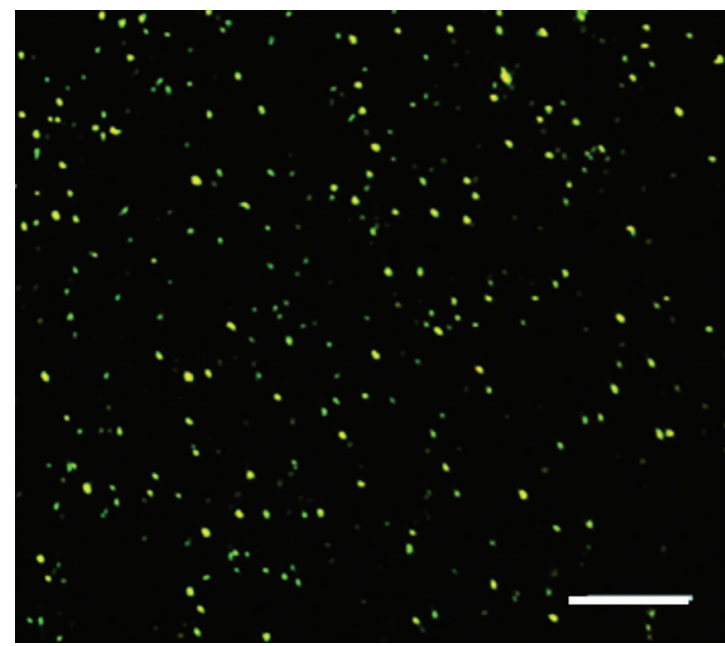

(c)

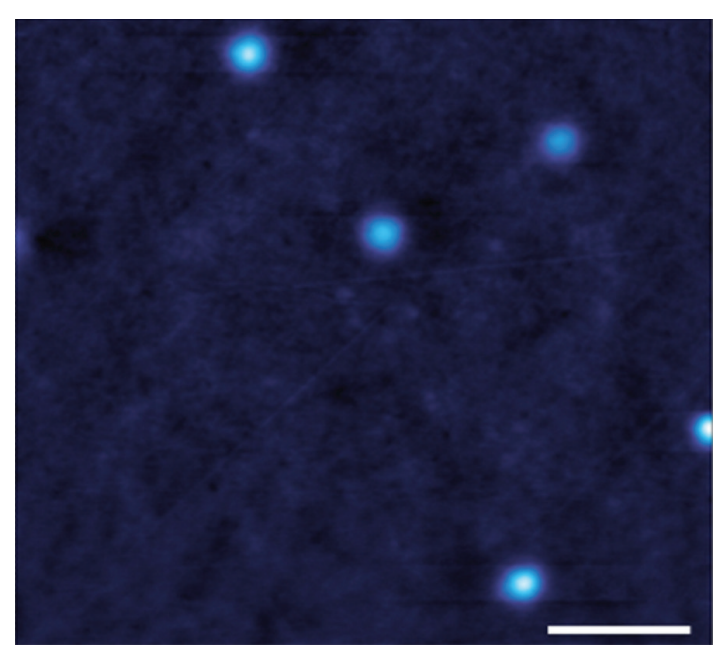

(b)

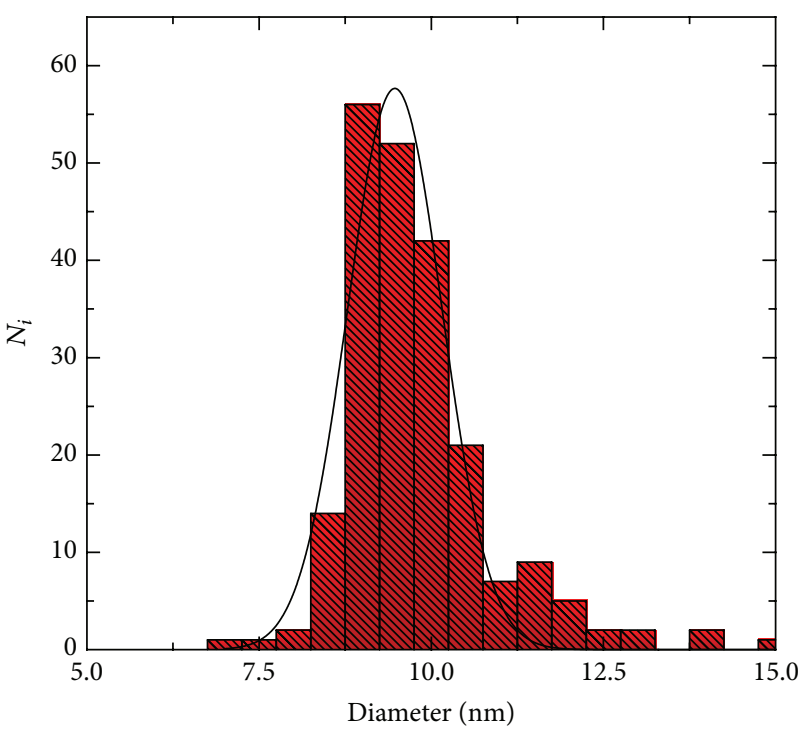

(d)

FiguRE 5: (a) Luminescence spectra $\left(\lambda_{\text {exc }}=450 \mathrm{~nm}\right)$ of the pristine Ag-NCs (black line) and of the photo-cross-linked Ag-NCs before (red circles) and after irradiation at $420 \mathrm{~nm}$ for $45 \mathrm{~min}$ (blue circles); (b) laser scanning fluorescence image (scale bar $=5 \mu \mathrm{m}$ ) and (c) AFM image $($ scale bar $=500 \mathrm{~nm})$ of photo-cross-linked Ag-NCs with the relative $(\mathrm{d})$ size distribution histogram.

information [35], the mechanism of vinylpyrrolidone photopolymerization is based on radical species whose formation can be assisted by the solvent and the metal cores. The irradiation conditions and the oligomer concentration were optimized in order to keep the system as a suspension, despite the increase of the sample viscosity. The luminescence spectrum of the UV-photo-cross-linked Ag-NCs presented a shape similar to the one recorded before the irradiation treatment (Figure 5(a)). Furthermore, the emission resulted to be much more stable; indeed, intensity or shape modifications were not observed under Vis irradiation over the same time period that causes the complete quenching of the emission of the untreated Ag-NCs (Figures 4 and 5(a)). The luminescence of a diluted suspension of Ag-NCs after PVP photo-cross-linking was also observed by the use of a confocal laser scanning microscope (Figure 5(b)). The images recorded upon excitation at $400 \mathrm{~nm}$ showed bright spots of diffraction-limited size $(\approx 0.3 \mu \mathrm{m})$ attributed to the emission of the silver nanoparticles. Furthermore, the data showed that the luminescence intensity did not decrease after up to $30 \mathrm{~min}$ of exposure to the excitation beam $(\lambda=$ $400 \mathrm{~nm}$ ), demonstrating the high photostability of the UVcured nanoparticles. This enhanced photostability might be related to the increase of viscosity of the polymeric shell, 
which hardens the NCs environment making the Ostwald ripening processes less efficient.

To investigate the effects of UV curing on the morphology/size of the metal cores, the luminescence spectra of the NCs were further investigated. Indeed, the convolution of the luminescence spectrum of Ag-NCs after photo-crosslinking of the polymeric shell could be reproduced by three Gaussian functions (Figure 3(b)), whose center values were the same, within the experimental errors, as the ones used to convolute the untreated samples; only a change of the relative weights of the replica was observed upon UV irradiation. These observations suggested that during the phototreatment no significant changes in size and shape of the silver nuclei occurred, since the optical properties are very sensitive to the morphological changes of the particles. AFM imaging (Figure 5(c)) indicates that the phototreated clusters maintained the spherical shape while their mean size distribution (measured as total height) shifted toward lower values (Figure 5(d)). This modification (in the order of 15$20 \%$ ) could be attributed to the shrinking of the polymeric shell induced by the photo-cross-linking process, thus confirming that the photomanipulation of the capping shell is responsible for the inhibition of the ripening processes.

\section{Conclusions}

In conclusion, $\mathrm{Ag}$-NCs were prepared in $\mathrm{CHCl}_{3}$ and their growth was controlled by means of PVP oligomers, which are well-known photo-cross-linkable units. This latter process was used to freeze the NCs morphology and optical behavior; indeed, by selective and controlled UV irradiation, the polymeric capping agent was successfully photo-cross-linked and stiffened in order to slow down or reduce Ostwald ripening processes responsible for NCs growth and morphological changes under Vis irradiation. Luminescence measurements together with TEM and AFM imaging allowed establishing the fact that the UV treatment did not affect the morphology of the metal cores. Through this low-cost and clean procedure, an important enhancement in the particle stability was reached, thus extending the usefulness of luminescent silver nanocrystals as imaging tools.

\section{Disclosure}

The present address for Silvia Rinaldi is ICRM-CNR, Istituto di Chimica del Riconoscimento Molecolare, Via Mario Bianco 9, 20131 Milan, Italy.

\section{Competing Interests}

The authors declare that they have no competing interests.

\section{Acknowledgments}

This work was supported by Università di Perugia and Ministero per l'Università e la Ricerca Scientifica e Tecnologica (Rome). Luigi Tarpani acknowledges the support of "Fondazione Cassa di Risparmio di Perugia" for funding.

\section{References}

[1] L. M. Liz-Marzán, "Tailoring surface plasmons through the morphology and assembly of metal nanoparticles," Langmuir, vol. 22, no. 1, pp. 32-41, 2006.

[2] V. Giannini, A. I. Fernández-Domínguez, S. C. Heck, and S. A. Maier, "Plasmonic nanoantennas: fundamentals and their use in controlling the radiative properties of nanoemitters," Chemical Reviews, vol. 111, no. 6, pp. 3888-3912, 2011.

[3] G. Kawamura, M. Nogami, and A. Matsuda, "Shape-controlled metal nanoparticles and their assemblies with optical functionalities," Journal of Nanomaterials, vol. 2013, Article ID 631350, 17 pages, 2013.

[4] P. K. Jain, X. Huang, I. H. El-Sayed, and M. A. El-Sayed, "Review of some interesting surface plasmon resonance-enhanced properties of noble metal nanoparticles and their applications to biosystems," Plasmonics, vol. 2, no. 3, pp. 107-118, 2007.

[5] V. Amendola, O. M. Bakr, and F. Stellacci, "A study of the surface plasmon resonance of silver nanoparticles by the discrete dipole approximation method: effect of shape, size, structure, and assembly," Plasmonics, vol. 5, no. 1, pp. 85-97, 2010.

[6] I. Díez and R. H. A. Ras, "Fluorescent silver nanoclusters," Nanoscale, vol. 3, no. 5, pp. 1963-1970, 2011.

[7] S. Choi, R. M. Dickson, and J. Yu, "Developing luminescent silver nanodots for biological applications," Chemical Society Reviews, vol. 41, no. 5, pp. 1867-1891, 2012.

[8] Z. Luo, K. Zheng, and J. Xie, "Engineering ultrasmall watersoluble gold and silver nanoclusters for biomedical applications," Chemical Communications, vol. 50, no. 40, pp. 5143-5155, 2014.

[9] E. Coutino-Gonzalez, M. B. J. Roeffaers, B. Dieu et al., "Determination and optimization of the luminescence external quantum efficiency of silver-clusters zeolite composites," The Journal of Physical Chemistry C, vol. 117, no. 14, pp. 6998-7004, 2013.

[10] X. Yuan, Y. Tay, X. Dou, Z. Luo, D. T. Leong, and J. Xie, "Glutathione-protected silver nanoclusters as cysteine-selective fluorometric and colorimetric probe," Analytical Chemistry, vol. 85, no. 3, pp. 1913-1919, 2013.

[11] S. Ghosh, U. Anand, and S. Mukherjee, "Luminescent silver nanoclusters acting as a label-free photoswitch in metal ion sensing," Analytical Chemistry, vol. 86, no. 6, pp. 3188-3194, 2014.

[12] E. Fortunati, L. Latterini, S. Rinaldi, J. M. Kenny, and I. Armentano, "PLGA/Ag nanocomposites: in vitro degradation study and silver ion release," Journal of Materials Science: Materials in Medicine, vol. 22, no. 12, pp. 2735-2744, 2011.

[13] H. Wei and H. Xu, "Hot spots in different metal nanostructures for plasmon-enhanced Raman spectroscopy," Nanoscale, vol. 5, no. 22, pp. 10794-10805, 2013.

[14] I. Chakraborty, S. Bag, U. Landman, and T. Pradeep, "Atomically precise silver clusters as new SERS substrates," The Journal of Physical Chemistry Letters, vol. 4, no. 16, pp. 2769-2773, 2013.

[15] Y. Zhang, C. Zhu, L. Zhang et al., "DNA-templated silver nanoclusters for multiplexed fluorescent DNA detection," Small, vol. 11, no. 12, pp. 1385-1389, 2015.

[16] R. Jin, Y. C. Cao, E. Hao, G. S. Métraux, G. C. Schatz, and C. A. Mirkin, "Controlling anisotropic nanoparticle growth through plasmon excitation," Nature, vol. 425, no. 6957, pp. 487-490, 2003.

[17] G. Baraldi, J. Gonzalo, J. Solis, and J. Siegel, "Reorganizing and shaping of embedded near-coalescence silver nanoparticles 
with off-resonance femtosecond laser pulses," Nanotechnology, vol. 24, no. 25, Article ID 255301, 2013.

[18] J. M. Kim, S. H. Sohn, N. S. Han, S. M. Park, J. Kim, and J. K. Song, "Blue luminescence of dendrimer-encapsulated gold nanoclusters," ChemPhysChem, vol. 15, no. 14, pp. 2917-2921, 2014.

[19] X. Liu, F. Wang, A. Niazov-Elkan, W. Guo, and I. Willner, "Probing biocatalytic transformations with luminescent DNA/silver nanoclusters," Nano Letters, vol. 13, no. 1, pp. 309-314, 2013.

[20] M. R. Carro Temboury, V. Paolucci, E. N. Hooley, L. Latterini, and T. Vosch, "Probing DNA-stabilized fluorescent silver nanocluster spectral heterogeneity by time-correlated single photon counting," Analyst, vol. 141, no. 1, pp. 123-130, 2016.

[21] G. De Cremer, B. F. Sels, J.-I. Hotta et al., "Optical encoding of silver zeolite microcarriers," Advanced Materials, vol. 22, no. 9, pp. 957-960, 2010.

[22] M. Periolatto, F. Ferrero, and C. Vineis, "Antimicrobial chitosan finish of cotton and silk fabrics by UV-curing with 2-hydroxy2-methylphenylpropane-1-one," Carbohydrate Polymers, vol. 88, no. 1, pp. 201-205, 2012.

[23] I. Struzyńska-Piron, J. Loccufier, L. Vanmaele, and I. F. J. Vankelecom, "Synthesis of solvent stable polymeric membranes via UV depth-curing," Chemical Communications, vol. 49, no. 98, pp. 11494-11496, 2013.

[24] M. Sangermano, N. Razza, and J. V. Crivello, "Cationic UVcuring: technology and applications," Macromolecular Materials and Engineering, vol. 299, no. 7, pp. 775-793, 2014.

[25] M. Amelia, D. Zoppitelli, C. Roscini, and L. Latterini, "Luminescence enhancement of organic nanoparticles induced by photocrosslinking," ChemPhysChem, vol. 11, no. 14, pp. 30893092, 2010.

[26] L. Tarpani and L. Latterini, "Driving the interactions between organic nanoparticles and phospolipidic membranes by an easy treatment of the surface stabilizer," Langmuir, vol. 29, no. 36, pp. 11405-11412, 2013.

[27] I. Pastoriza-Santos and L. M. Liz-Marzán, "Synthesis of Silver Nanoprisms in DMF," Nano Letters, vol. 2, no. 8, pp. 903-905, 2002.

[28] A. Sarkar, S. Kapoor, and T. Mukherjee, "Preparation, characterization, and surface modification of silver nanoparticles in formamide," The Journal of Physical Chemistry B, vol. 109, no. 16, pp. 7698-7704, 2005.

[29] C. E. Hoppe, M. Lazzari, I. Pardiñas-Blanco, and M. A. LópezQuintela, "One-step synthesis of gold and silver hydrosols using poly(N-vinyl-2- pyrrolidone) as a reducing agent," Langmuir, vol. 22, no. 16, pp. 7027-7034, 2006.

[30] B. Wiley, Y. Sun, B. Mayers, and Y. Xia, "Shape-controlled synthesis of metal nanostructures: the case of silver," Chemistry-A European Journal, vol. 11, no. 2, pp. 454-463, 2005.

[31] J. Jiu, K. Murai, D. Kim, K. Kim, and K. Suganuma, "Preparation of Ag nanorods with high yield by polyol process," Materials Chemistry and Physics, vol. 114, no. 1, pp. 333-338, 2009.

[32] M. K. Corbierre, N. S. Cameron, M. Sutton, K. Laaziri, and R. B. Lennox, "Gold nanoparticle/polymer nanocomposites: dispersion of nanoparticles as a function of capping agent molecular weight and grafting density," Langmuir, vol. 21, no. 13, pp. 6063-6072, 2005.

[33] J. J. Mock, M. Barbic, D. R. Smith, D. A. Schultz, and S. Schultz, "Shape effects in plasmon resonance of individual colloidal silver nanoparticles," The Journal of Chemical Physics, vol. 116, no. 15 , pp. $6755-6759,2002$.
[34] F. Haaf, A. Sanner, and F. Straub, "Polymers of n-vinylpyrrolidone: synthesis, characterization and uses," Polymer Journal, vol. 17, no. 1, pp. 143-152, 1984.

[35] M. Stach, I. Lacík, D. Chorvát Jr. et al., "Propagation rate coefficient for radical polymerization of $\mathrm{N}$-vinyl pyrrolidone in aqueous solution obtained by PLP-SEC", Macromolecules, vol. 41, no. 14, pp. 5174-5185, 2008. 

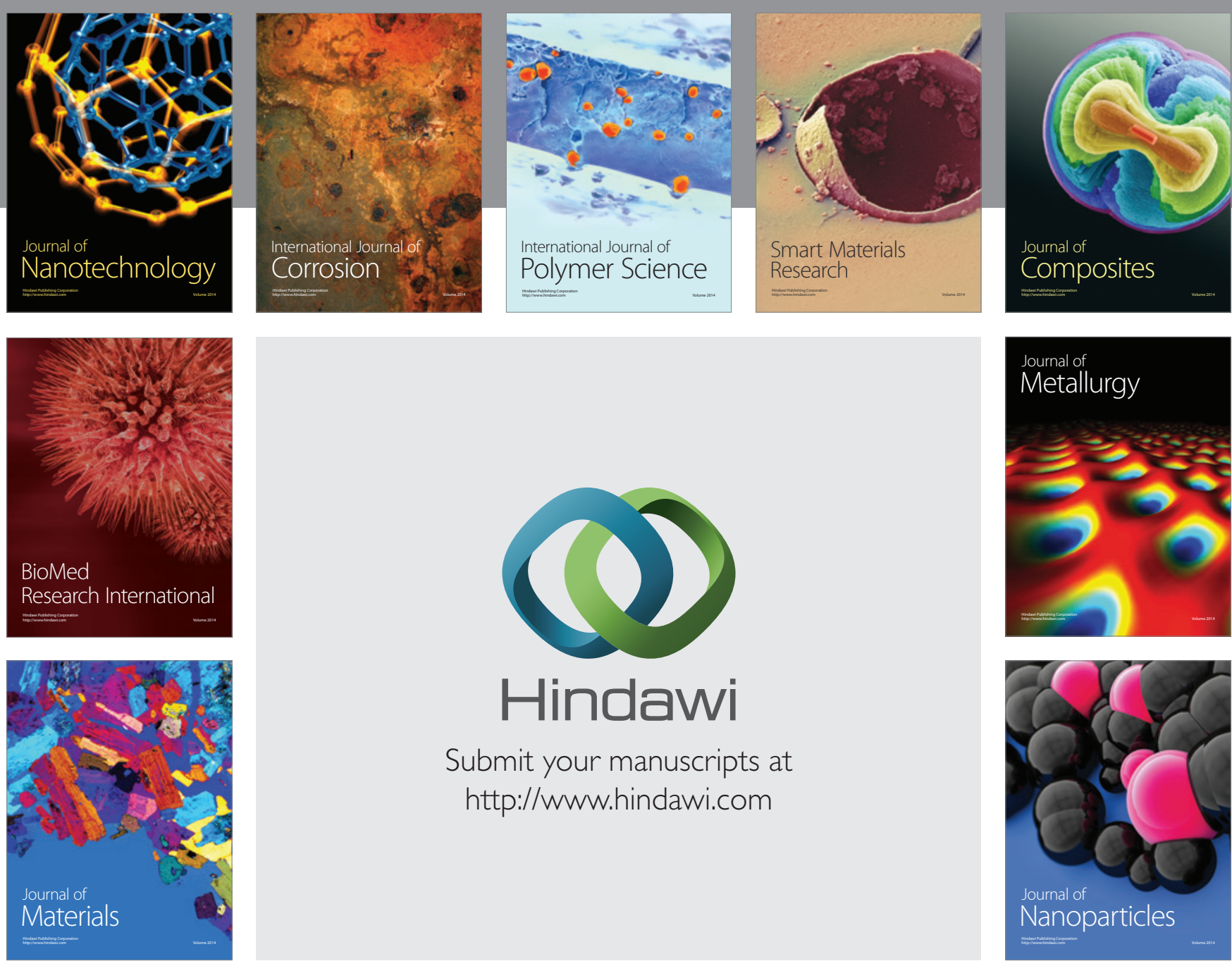

\section{Hindawi}

Submit your manuscripts at

http://www.hindawi.com

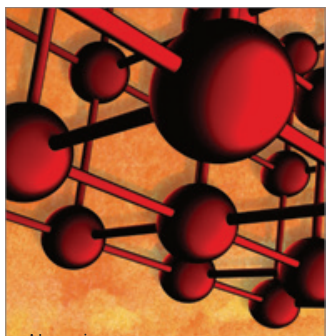

Materials Science and Engineering
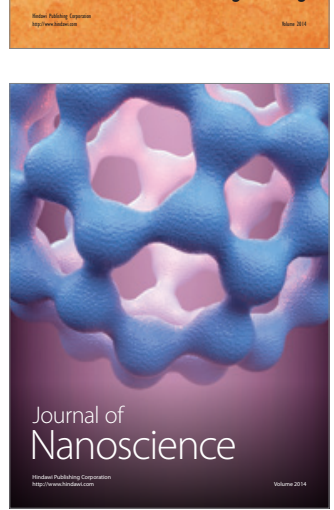
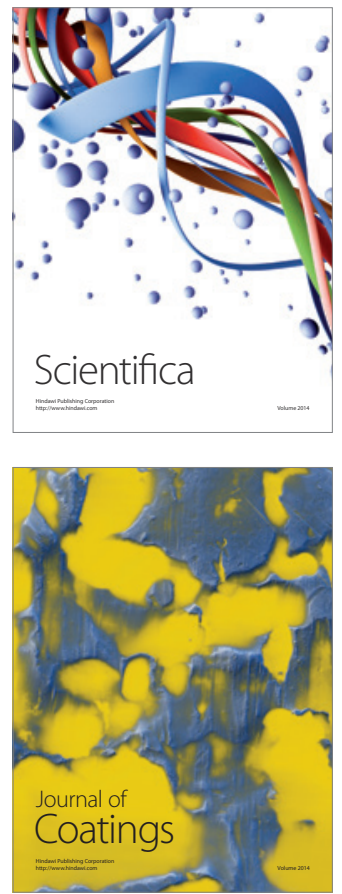
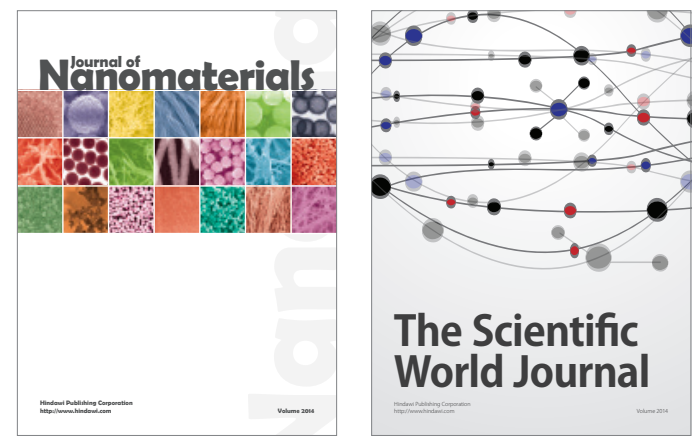

The Scientific World Journal
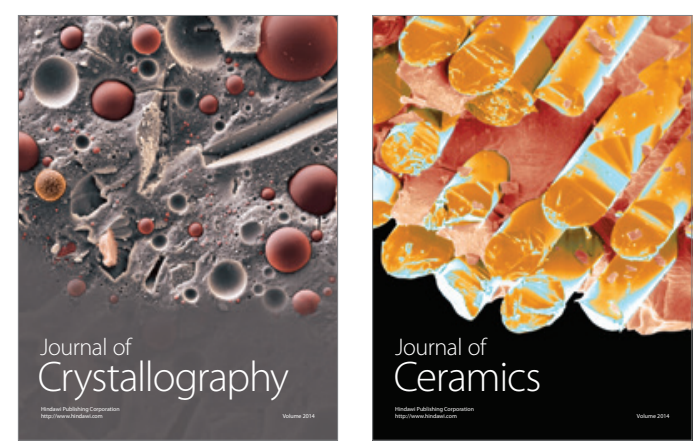
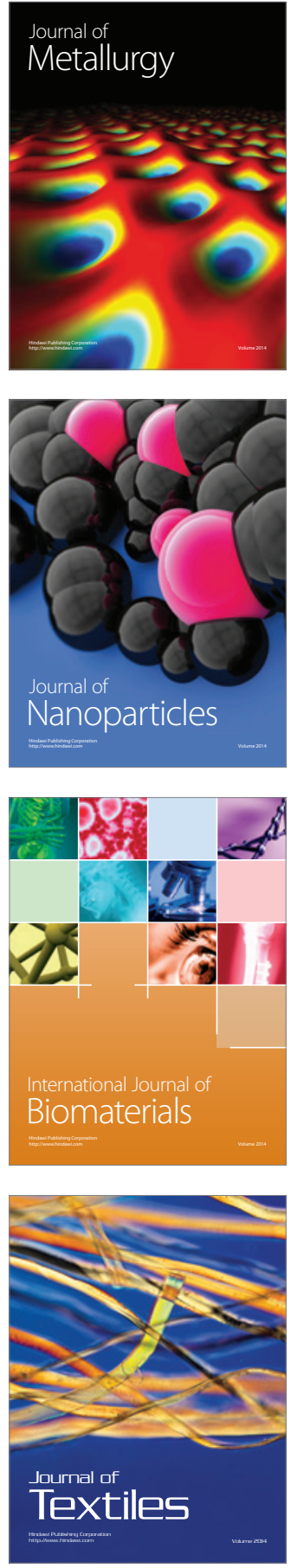